\title{
GLAST Status and Application to Microquasars
}

\author{
Richard Dubois*独 \\ Stanford Linear Accelerator Center, Menlo Park CA, USA \\ E-mail: richardeslac.stanford.edu
}

for the LAT Collaboration

The Gamma-ray Large Area Space Telescope (GLAST) is a next generation high energy gammaray observatory due for launch in Fall 2007. The primary instrument is the Large Area Telescope (LAT), which will measure gamma-ray flux and spectra from $20 \mathrm{MeV}$ to $>300 \mathrm{GeV}$ and is a successor to the highly successful EGRET experiment on CGRO. The LAT will have better angular resolution, greater effective area, wider field of view and broader energy coverage than any previous experiment in this energy range. An overview of the LAT instrument design and construction is presented which includes performance estimates with particular emphasis on how these apply to strudies of microquasars. The nature and quality of the data that will be provided by the LAT is described with results from recent detailed simulations that illustrate the potential of the LAT to observe gamma ray variability and spectra.

VI Microquasar Workshop: Microquasars and Beyond

September 18-22, 2006

Como, Italy

\footnotetext{
* Speaker.

${ }^{\dagger}$ This work is supported by Stanford University and the Stanford Linear Accelerator Center (SLAC) under DoE contract number DE-AC03-76SFO0515.
} 


\section{Introduction}

GLAST is a next generation high-energy gamma-ray observatory designed for making observations of celestial gamma ray sources in the energy band extending from $20 \mathrm{MeV}$ to more than $300 \mathrm{GeV}$. It follows in the footsteps of the Compton Gamma Ray Observatory EGRET experiment, which was operational between 1991-1999. The GLAST Mission is part of NASA's Office of Space and Science Strategic Plan, with launch anticipated in 2007. The principal instrument of the GLAST mission is the Large Area Telescope (LAT) that is being developed jointly by NASA and the US Dept. of Energy (DOE) and is supported by an international collaboration of 26 institutions lead by Stanford University.

The GLAST LAT is a high-energy pair conversion telescope that has been under development for over 10 years with support from NASA, DOE and international partners. It consists of a precision converter-tracker, CsI hodoscopic calorimeter, plastic scintillator anticoincidence system and a data acquisition system. The design is modular with a $4 \times 4$ array of identical tracker and calorimeter modules. The modules are approximately $38 \times 38 \mathrm{~cm}$. Figure 1 shows the LAT instrument concept.

The LAT science instrument consists of an Anti Coincidence Detector

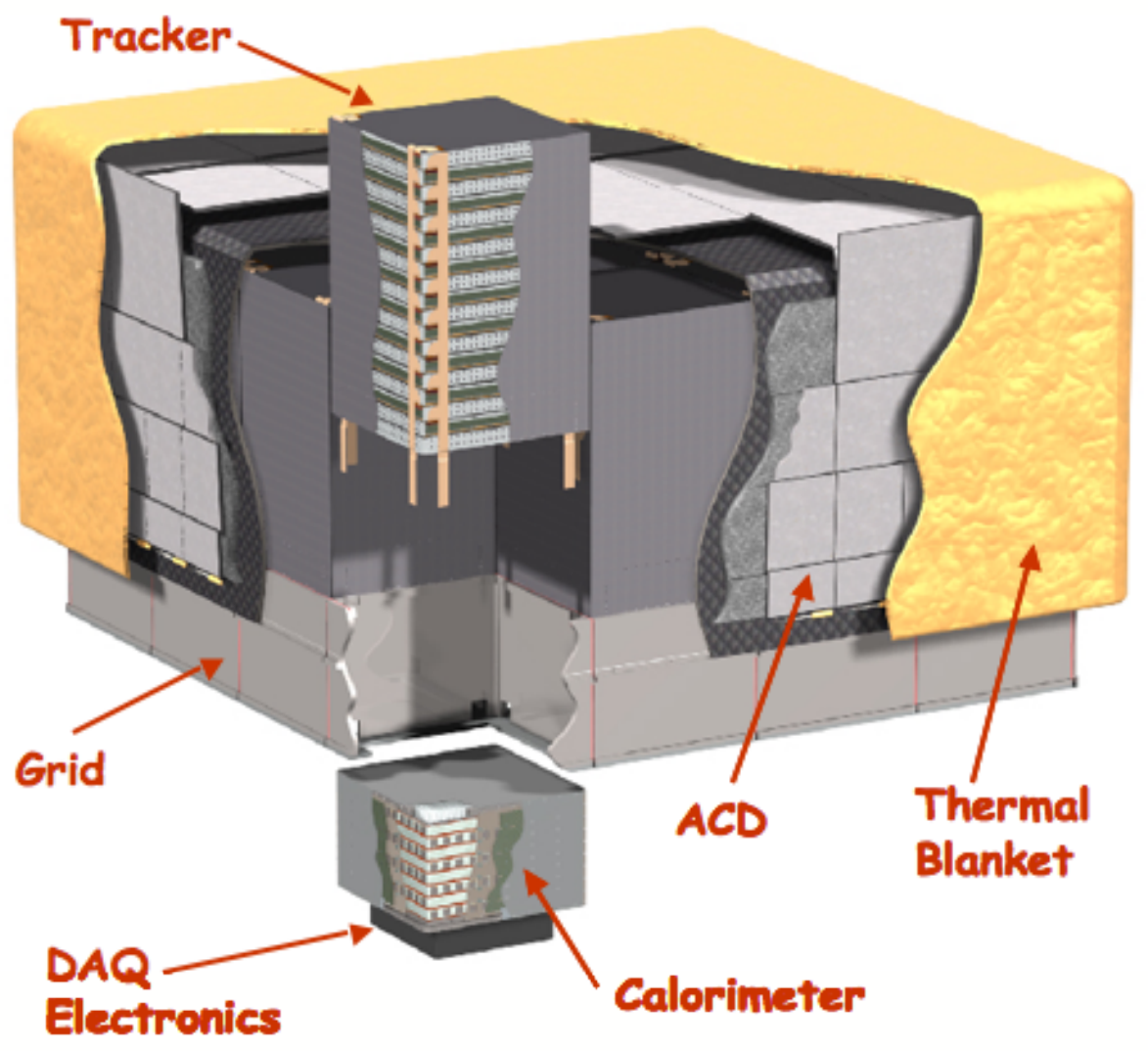

Figure 1: Large Area Telescope. Composed of 16 modules containing Tracker and Calorimeter elements, all surrounded by a scintillator anti-coincidence shield.

(ACD), a silicon-strip detector Tracker (TKR), a hodoscopic CsI Calorimeter (CAL), and a Trigger and Data Flow system (T\&DF). The principal purpose of the LAT is to measure the incidence direction, energy and time of cosmic gamma rays while rejecting background from charged 
cosmic rays and atmospheric albedo gamma rays and particles. The data, filtered by onboard software triggers, are streamed to the spacecraft for data storage and subsequent transmittal to ground-based analysis centers. The Tracker provides the principal trigger for the LAT, converts the gamma rays into electron-positron pairs, and measures the direction of the incident gamma ray from the charged-particle tracks. The first 12 layers of converter are about $3 \% \mathrm{X}_{0}$, while the next 4 layers (back) are about $18 \%$. This was done to optimize efficiency for photons interacting near the calorimeter. The tracker is crucial in the first levels of background rejection for providing track information to extrapolate cosmic-ray tracks to the ACD scintillator tiles, and it is important for further levels of background analysis due to its capability to provide highly detailed track patterns in each event.

In normal operations the LAT will continually scan the sky, obtaining essentially complete sky coverage every 3 hours (two orbits). This uniformity of sky coverage together with the large effective area and good angular resolution should permit many advances in the study of microquasars in the GeV range. The performance properties of the LAT are summarized in Table 1. The most current LAT performance specifications are kept online[1].

Table 1: LAT Energy and Angular Resolution

$\begin{array}{cc}\text { Energy Resolution } & \approx 10 \%(\approx 5 \% \text { off axis }) \\ \text { PSF }(68 \%) \text { at } 100 \mathrm{MeV} & 3.5^{0} \text { front; } 5^{0} \text { total } \\ \text { PSF }(68 \%) \text { at } 10 \mathrm{GeV} & 0.1^{0} \\ \text { Field of View } & 2.4 \mathrm{sr} \\ \text { Source Sensitivity }(>100 \mathrm{MeV}) & 3 \times 10^{-9} \mathrm{~cm}^{-2} \mathrm{~s}^{-1}\end{array}$

Point Source Sensitivity (> $100 \mathrm{MeV}) \quad 3 \times 10^{-9} \mathrm{~cm}^{-2} \mathrm{~s}^{-1}$

\section{Feasibility Study for Microquasar Observations}

GLAST's Data Challenge 2 (DC2) provided a detailed simulation of the sky and the LAT's response. $5 \mathrm{X}$-ray binaries were included with flux and spectra from EGRET measurements and known orbital periods. Fifty-five days of simulated orbit were created using a full GEANT4 simulation of LAT response, with full time dependence in the simulations for AGN, solar flares, GRBs. It took some 200k CPU hours to produce this rendition of the sky. Modeling of the XRB's was very simple, with single power law energy spectra and full modulation of the orbital dependence.

For initial studies into GLAST's capabilities in observing microquasars we have concentrated on the two which have been measured in TeV gamma rays, by HESS[3] and MAGIC[4]. LS 5039 is near the galactic center and has two other nearby sources as well as a significant galactic diffuse background. On the other hand, LSI +61 303 is more isolated and in a lower diffuse background. In addition, their orbital periods differ by almost an order of magnitude. These provide good examples of the extremes of observations the LAT will be called on to make. We will investigate the LAT's ability to determine the flux, spectral index and orbital time variability in this pilot study.

\subsection{Full simulation 55 day orbit data}

LSI +61 303 is shown to be isolated and in a low background region as shown in Figure 2. It is straightforward to use a likelihood fit on this region to include the galactic diffuse background 
as well as a point source with a single power law spectrum. Such a fit results in a spectral index of $2.21 \pm 0.07$, compared to the simulated value of 2.19 . A fit to the $1 \mathrm{vs} b$ distribution yields a one-sigma error of about $0.02^{0}$ in each variable.
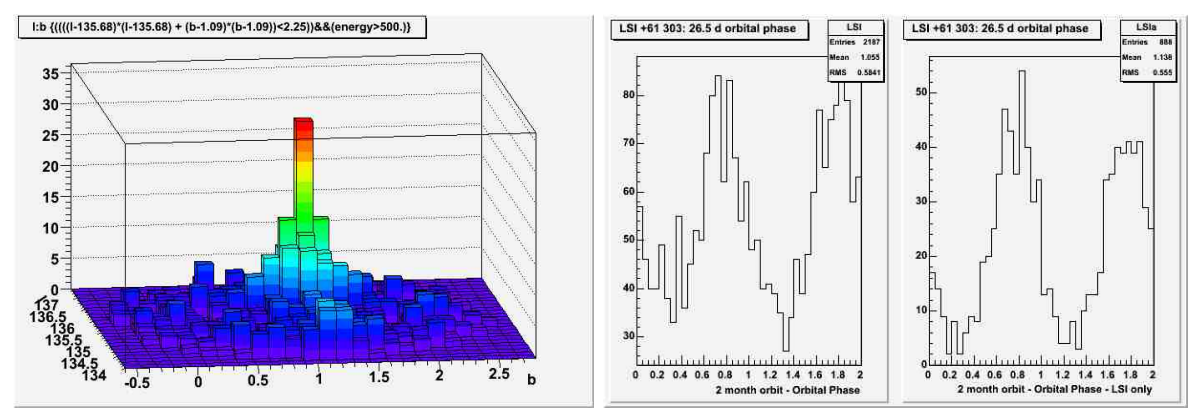

Figure 2: Left: Galactic coordinates $l$ vs $b$ for LSI +61303 for 55 days' orbit data. No other known sources are within a few degrees, and galactic diffuse background is low. LSI +61 303 orbital phase for E > 100 MeV. Middle panel shows all photons. Right panel shows photons from LSI +61 303 only.

Figure 2 also shows the orbital period phase for two periods for both all photons around LSI +61303 , and the from the source alone. The full modulation is clearly seen, and also is plainly visible for the all photon case, demonstrating the ability to detect the 29.5 day period.

Things are not so rosy for LS 5039, as shown in Figure 3. The background to noise ratio is about 15:1, so extracting the source photons will be very difficult. An attempt was made to use the PERIOD[2] package, utilizing Lomb-Scargle periodigrams, to find the orbital period: for the LS 5039 photons alone, the orbital period was found to within 5\%; for the full photon sample, the process did not converge.
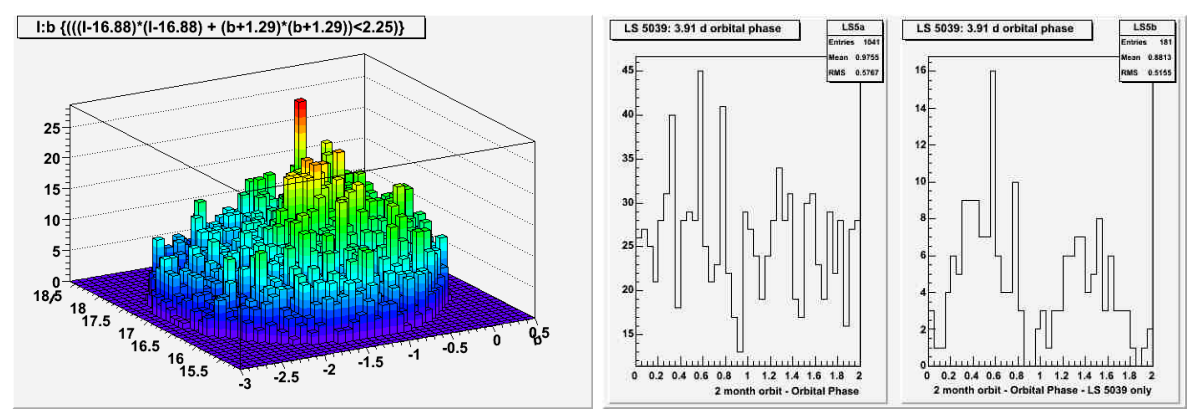

Figure 3: Left: Galactic coordinates $l$ vs $b$ for LS 5039 for 55 days' orbit data. There is no sign of LS 5039 at the generated source location $\left(16.9^{0},-1.3^{0}\right)$. Orbital phase for E $>100 \mathrm{MeV}$. Middle panel shows all photons. Right panel shows photons from LS 5039 only.

\subsection{Fast simulation one year orbit data}

A full year of orbit data was simulation using a parametrization of the instrument response and effective area in order to investigate how much more data improves the situation for LS 5039: we will take advantage of the spectrum being harder than that of the diffuse and that at higher energies the photon directions are much better determined. This is illustrated in Figure 4. In this case, the source is easily found and the 3.91 day orbital period has become apparent. A fit to the $1 \mathrm{vs} \mathrm{b}$ distribution yields an error of about $0.05^{0}$ in each variable. 

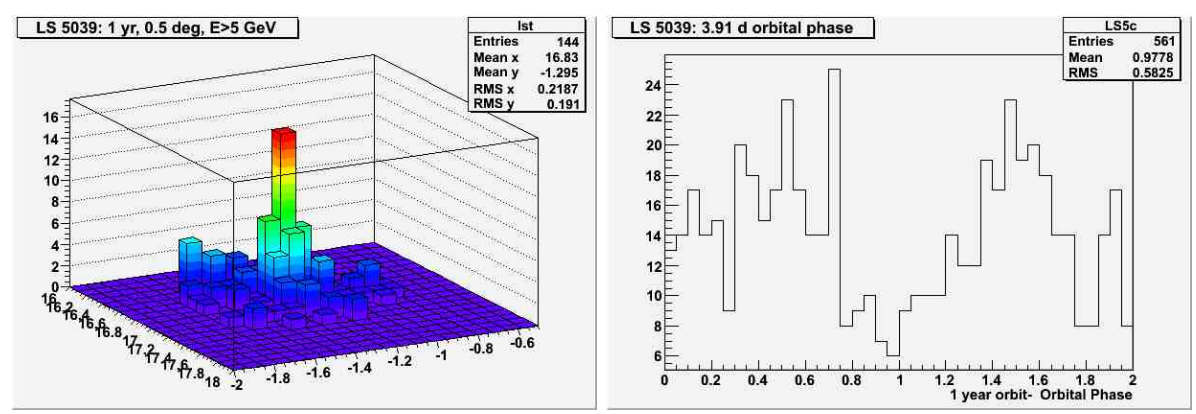

Figure 4: Left: Galactic coordinates $l$ vs $b$ for LS 5039 for one year's' orbit data, E > 2 GeV. LS 5039 is now quite evident. Orbital phase for $\mathrm{E}>2 \mathrm{GeV}$. MIddle panel shows all photons. Right panel shows photons from LS 5039 only.

\section{Conclusions}

GLAST will launch in late 2007 and provide a unique view of the gamma ray sky over the 0.1-200 GeV energy range. The large field of view and survey mode of the LAT will allow us to monitor all the microquasar candidates on a continuous basis.

For isolated sources, we will have no difficulty measuring the spectral and orbital period properties for sources with fluxes like those of LSI +61 303 and LS $5039\left(\approx 10^{-7} \mathrm{ph} \mathrm{s}^{-1} \mathrm{~cm}^{-2}\right)$. For sources living in confused regions of the sky, like LS 5039, we will need to integrate longer and make harder energy cuts to bring the signal out above background. A better source localisation could in principle be achieved with a likelihood analysis that took into account that the PSF is a strong function of photon energy.

Prior to launch we plan to extend our full simulations to a full year of orbit data and develop the machinery to observe all the microquasar candidates. We will also investigate our ability to detect time variabilities other than orbital and develop strategies for multi-wavelength campaigns.

\section{References}

[1] S.Ritz et al, LAT Performance Specifications, http://www-glast.slac.stanford.edu/software/IS/glast_lat_performance.htm

[2] PERIOD, StarLink Project, http://www.starlink.rl.ac.uk/Bulletin/96sep/a25.html

[3] Aharonian et al, astro-ph 0607192

[4] Alberts et al, Science, 2006 (1771) 312 